\title{
Negative refraction in Weyl semimetals
}

\author{
M. Shoufie Ukhtary, ${ }^{*}$ Ahmad R. T. Nugraha, and Riichiro Saito \\ Department of Physics, Tohoku University, Sendai 980-8578, Japan
}

(Dated: September 19, 2018)

\begin{abstract}
We theoretically propose that Weyl semimetals may exhibit negative refraction at some frequencies close to the plasmon frequency, allowing transverse magnetic (TM) electromagnetic waves with frequencies smaller than the plasmon frequency to propagate in the Weyl semimetals. The idea is justified by the calculation of reflection spectra, in which negative refractive index at such frequencies gives physically correct spectra. In this case, a TM electromagnetic wave incident to the surface of the Weyl semimetal will be bent with a negative angle of refraction. We argue that the negative refractive index at the specified frequencies of the electromagnetic wave is required to conserve the energy of the wave, in which the incident energy should propagate away from the point of incidence.
\end{abstract}

\section{INTRODUCTION}

Negative refraction phenomenon has attracted many interests since its prediction by Veselago a half century ago. [1] Veselago predicted that if a material possesses simultaneous negative dielectric constant $(\varepsilon)$ and magnetic permeability $(\mu)$, it will give a negative refractive index. The negative refractive index will lead to some unusual properties of the light, such as negative refraction and reversed Doppler and Cherenkov effects. [1-6] By utilizing negative refraction, in which the light will be bent in an unusual way with an angle of refraction negative to the normal direction of the material surface, one may be able to construct a superlens whose resolution is smaller than the light wave length. $[2,3,6] \mathrm{A}$ better Cherenkov radiation detector can also be realized based on the material having a negative refractive index, which is useful in the field of accelerator physics. [7, 8] However, materials having simultaneous negative $\varepsilon$ and $\mu$ have not been found in nature so far.

To realize negative refraction, many researchers developed artificial structures that are called as metamaterials. [9-12] These structures usually contain an array of split ring resonators [6, 13-15] or dielectric photonic crystals with periodically modulated $\varepsilon$ and $\mu,[2,6,16]$ which are often complicated to fabricate. To overcome the difficulties, in this paper we predict that negative refraction can take place in a bulk Weyl semimetal (WSM) even without having negative $\mu$ and without constructing complicated structure. The WSM is a threedimensional material having a pair of Dirac cones separated in the $k$ space in its energy dispersion shown in Fig. 1(a). [17-21] An example of the WSM is pyrochlore $\left(\mathrm{Eu}_{2} \mathrm{Ir}_{2} \mathrm{O}_{7}\right) .[17,22]$ In each cone, the valence and conduction bands coincide at the so-called Weyl nodes. The presence of a pair of separated Dirac cones is the consequence of symmetry breaking in the WSM, which induces the Hall current, even without magnetic field. [1719] This phenomenon is known as the anomalous Hall effect, which is responsible for the tensor form of the di-

\footnotetext{
*shoufie@flex.phys.tohoku.ac.jp
}

electric function of the WSM $[17,23]$. In this work, we predict that the EM wave can propagate through WSM even though the frequency is smaller than plasmon frequency. This propagation requires the refractive index of WSM to be negative in order to conserve the energy, that will be shown in this paper.

\section{MODEL AND METHODS}

The electromagnetic response of WSM can be derived from the formula of action for the electromagnetic field. [19, 23, 24] Here, we will give brief derivation of the electromagnetic response of WSM represented by electric displacement vector $\mathbf{D}$. The more detailed derivation is given by Zyuzin and Burkov [25, 26] or Hosur and Qi. [27] The action of electromagnetic field is given by,

$$
S_{\theta}=-\frac{e^{2}}{8 \pi^{2} \hbar} \int d t d \mathbf{r} \partial_{\gamma} \theta \epsilon^{\gamma \nu \rho \eta} A_{\nu} \partial_{\rho} A_{\eta},
$$

where $A_{\nu}$ is electromagnetic potential, $\epsilon^{\gamma \nu \rho \eta}$ is the Levi-Civita tensor and each index $\gamma, \nu, \rho, \eta$ takes values $0,1,2,3$. The term $\theta$ is called the axion angle given by $\theta=2(\mathbf{b} \cdot \mathbf{r})$, where $\mathbf{b}$ is a wave vector separating the Weyl nodes [see Figure 1(a)]. The current density $j_{\nu}$ is given by varying the action with respect to electromagnetic potential,

$$
j_{\nu} \equiv \frac{\delta S_{\theta}}{\delta A_{\nu}}=\frac{e^{2}}{4 \pi^{2} \hbar} \partial_{\gamma} \theta \epsilon^{\gamma \nu \rho \eta} \partial_{\rho} A_{\eta}
$$

By writing $\mathbf{E}=-\left(\nabla A_{0}\right)-\partial_{0} \mathbf{A}$, Eq. (2) gives the Hall current $\mathbf{j}=\frac{e^{2}}{4 \pi^{2} \hbar} \nabla \theta \times \mathbf{E}$, which gives additional terms in $\mathbf{D}$ of the normal metals as the second term of Eq. (3). We can write the electric displacement vector as follows,

$$
\mathbf{D}=\varepsilon_{0} \varepsilon_{b}\left(1-\frac{\omega_{p}^{2}}{\omega^{2}}\right) \mathbf{E}+\frac{i e^{2}}{4 \pi^{2} \hbar \omega}(\nabla \theta) \times \mathbf{E},
$$

where $\omega_{p}$ is the plasmon frequency, $\varepsilon_{b}$ is the background dielectric constant. Hereafter, we consider a particular value of the dielectric constant, $\varepsilon_{b}=13$, which was measured in pyrochlore. [17, 22] The first term of Eq. (3) is 

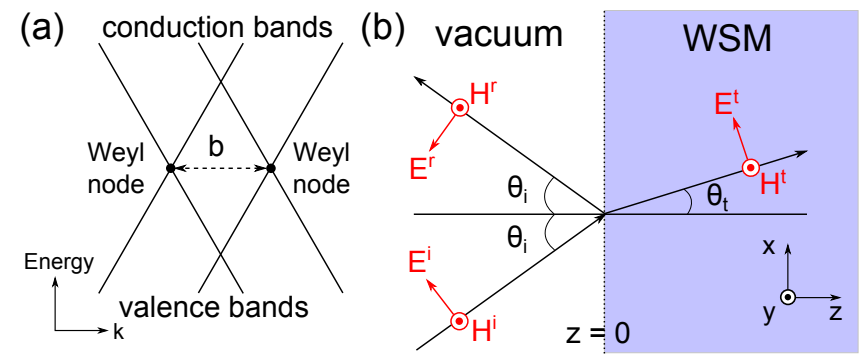

FIG. 1. (a) Schematic of energy dispersion of WSM showing a pair of Dirac cones with two Weyl nodes represented by dots, separated by the wave vector $b$. (b) A TM wave coming to $x y$ surface of WSM at angle $\theta_{i}$ and transmitted to WSM at angle $\theta_{t}$.

the Drude dielectric function, which is similar to normal metals (NMs). The appearance of Hall current without external magnetic field is known as anomalous Hall effect given by the second term of Eq. (3). The anomalous Hall current only depends on the structure of the electron dispersion of WSM represented by $\theta$. Due to the anomalous Hall effect, the dielectric tensor has non-zero off-diagonal terms, which can be written as

$$
\varepsilon=\left[\begin{array}{ccc}
\varepsilon_{1} & 0 & i \varepsilon_{2} \\
0 & \varepsilon_{1} & 0 \\
-i \varepsilon_{2} & 0 & \varepsilon_{1}
\end{array}\right]
$$

where we assume that $\mathbf{b}$ lies in the direction of $y, \mathbf{b}=b \hat{\mathbf{y}}$, and that $\varepsilon_{1}$ and $\varepsilon_{2}$ are expressed by

$$
\begin{aligned}
& \varepsilon_{1}=\varepsilon_{0} \varepsilon_{b}\left(1-\frac{1}{\Omega^{2}}\right), \\
& \varepsilon_{2}=\varepsilon_{0} \varepsilon_{b}\left(\frac{\Omega_{b}}{\Omega}\right),
\end{aligned}
$$

with $\Omega=\omega / \omega_{p}$ and $\Omega_{b}=e^{2} b /\left(2 \pi^{2} \varepsilon_{0} \varepsilon_{b} \hbar \omega_{p}\right)$ as dimensionless quantities. We take $\Omega_{b}=0.5$ as a fixed parameter throughout this paper, otherwise it will be mentioned.
Similar to NMs, in the WSM we have $\varepsilon_{1}>0\left(\varepsilon_{1}<0\right)$ if $\Omega>1(\Omega<1)$.

In order to calculate the reflection and transmission spectra of a bulk WSM, we will determine the refractive index of the WSM $\left(n_{w}\right)$. Suppose that we have a transverse magnetic (TM) wave incident at angle $\theta_{i}$ from vacuum to a WSM as shown in Fig. 1(b) where $E^{i}, E^{r}$ and $E^{t}\left(H^{i}, H^{r}\right.$ and $\left.H^{t}\right)$ are the incident, reflected and transmitted electric (magnetic) fields, respectively. The transmitted wave propagates toward positive $z$ direction inside WSM, while the reflected wave propagates toward negative $z$ direction. Due to the vanishing $\varepsilon_{x y}$ and $\varepsilon_{z y}$, the direction of electric field inside WSM does not rotate. By using Eq. (4), we can write down the equation $\mathbf{D}=\hat{\varepsilon} \mathbf{E}$ for the TM wave inside the WSM as follows,

$$
\left[\begin{array}{l}
D_{x}^{t} \\
D_{y}^{t} \\
D_{z}^{t}
\end{array}\right]=\left[\begin{array}{ccc}
\varepsilon_{1} & 0 & i \varepsilon_{2} \\
0 & \varepsilon_{1} & 0 \\
-i \varepsilon_{2} & 0 & \varepsilon_{1}
\end{array}\right]\left[\begin{array}{c}
E_{x}^{t} \\
0 \\
E_{z}^{t}
\end{array}\right]
$$

where $D^{t}$ and $E^{t}$ are the displacement and electric fields inside the WSM. From Maxwell's equations, we get a differential equation for the EM wave as follows;

$$
\nabla \times \nabla \times \mathbf{E}^{t}=-\nabla^{2} \mathbf{E}^{t}+\nabla\left(\nabla \cdot \mathbf{E}^{t}\right)=\omega^{2} \mu_{0} \mathbf{D}^{t} .
$$

Since the solutions of $\mathbf{E}^{t}$ and $\mathbf{D}^{t}$ are proportional to $\exp \left[i \omega n_{w} / c(\mathbf{s} \cdot \mathbf{r})\right]$, where $\mathbf{s}=\left(\sin \theta_{t}, 0, \cos \theta_{t}\right)$ is the unit wave vector, we can obtain from Eq. (8),

$$
\frac{1}{\mu_{0}}\left(\frac{n_{w}}{c}\right)\left[\mathbf{E}^{t}-\mathbf{s}\left(\mathbf{s} \cdot \mathbf{E}^{t}\right)\right]=\mathbf{D}^{t} .
$$

From Eqs. (7) and (9), we get the following relations,

$$
E_{x}^{t}=\frac{\varepsilon_{1} D_{x}^{t}-i \varepsilon_{2} D_{z}^{t}}{\varepsilon_{1}^{2}-\varepsilon_{2}^{2}}, \text { and } E_{z}^{t}=\frac{i \varepsilon_{2} D_{x}^{t}+\varepsilon_{1} D_{z}^{t}}{\varepsilon_{1}^{2}-\varepsilon_{2}^{2}} .
$$

Inserting Eq. (10) to Eq. (9), we obtain simultaneous equations of $E_{x}^{t}$ and $E_{z}^{t}$ as follows:

$$
\left[\begin{array}{cc}
\left(1-s_{x}^{2}\right) \varepsilon_{1}-i s_{x} s_{z} \varepsilon_{2}-\mu_{0}\left(\frac{c}{n_{w}}\right)^{2}\left(\varepsilon_{1}^{2}-\varepsilon_{2}^{2}\right) & -i\left(1-s_{x}^{2}\right) \varepsilon_{2}-s_{x} s_{z} \varepsilon_{1} \\
i\left(1-s_{z}^{2}\right) \varepsilon_{2}-s_{x} s_{z} \varepsilon_{1} & \left(1-s_{z}^{2}\right) \varepsilon_{1}+i s_{x} s_{z} \varepsilon_{2}-\mu_{0}\left(\frac{c}{n_{w}}\right)^{2}\left(\varepsilon_{1}^{2}-\varepsilon_{2}^{2}\right)
\end{array}\right]\left[\begin{array}{l}
E_{x}^{t} \\
E_{z}^{t}
\end{array}\right]=0
$$

In order to have nontrivial solutions of $\mathbf{E}^{t}$, the determinant of the $2 \times 2$ matrix in Eq. (11) should vanish:

$$
\frac{\mu_{0} c^{2}\left(\varepsilon_{1}^{2}-\varepsilon_{2}^{2}\right)}{n_{w}^{4}}\left[-n_{w}^{2} \varepsilon_{1}+c^{2} \mu_{0}\left(\varepsilon_{1}^{2}-\varepsilon_{2}^{2}\right)\right]=0 .
$$

from which, we obtain $n_{w}$,

$$
n_{w}= \pm c \sqrt{\mu_{0}\left(\varepsilon_{1}^{2}-\varepsilon_{2}^{2}\right) / \varepsilon_{1}} \equiv n_{w}^{ \pm}
$$

where the $n_{w}^{+}\left(n_{w}^{-}\right)$solution corresponds to the positive (negative) wave vector inside the WSM. If we put $\varepsilon_{2}=0$ in Eq. (13), we can obtain the refractive index of NM.

In Fig. 2(a) and (b) we plot $n_{w}$ as a function of $\Omega$ for the positive solution of Eq. (13) [Fig. 2(a)] and the negative solution of Eq. (13) [Fig. 2(b)]. The solid and the dashed lines correspond to the real and imaginary parts of $n_{w}$, respectively. It is noted that $n_{w}^{ \pm}$at each frequency is either purely real or purely imaginary, because we ne- 

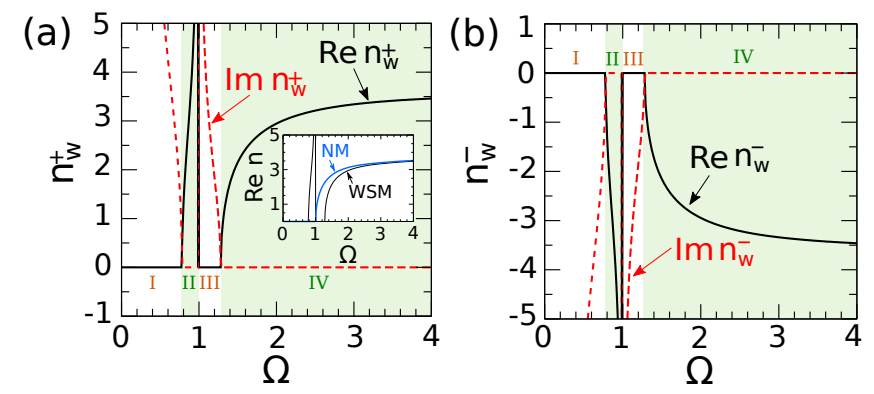

(c)

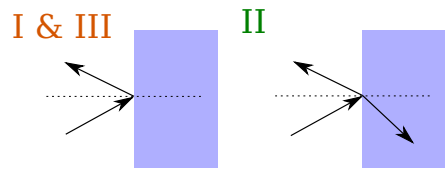

IV

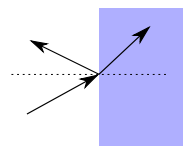

FIG. 2. (a) The refractive index of WSM for TM wave $\left(n_{w}\right)$ as a function of $\Omega$ for the positive solution of Eq. (13) $\left(n_{w}^{+}\right)$. Solid and dashed lines are the real and imaginary parts of $n_{w}^{+}$, respectively. We use $\Omega_{b}=0.5$ for the WSM. The plot is divided into four regions. Inset: The real part of refractive index $(n)$ for the WSM compared with a normal metal (NM). (b) The refractive index of WSM for TM wave $\left(n_{w}\right)$ as a function of $\Omega$ for the negative solution of Eq. (13) $\left(n_{w}^{-}\right)$. (c) Schematics of EM wave propagations to the WSM for all the four regions of panel (a) and (b).

glect the effects of the impurity and scattering of charge in Eq. (5). Therefore, the wave vector $\omega n_{w}^{ \pm} / c$ can be either real or imaginary depending on $n_{w}^{ \pm}$. The real (imaginary) wave vector represents a propagating (decaying) wave.

Here we divide our results into four regions as shown in Fig. 2(c): region I $\left(0 \leq \Omega \leq \Omega_{-}\right)$, region II $\left(\Omega_{-} \leq \Omega \leq 1\right)$, region III $\left(1 \leq \Omega \leq \Omega_{+}\right)$and region IV $\left(\Omega_{+} \leq \Omega\right)$, where $\Omega_{ \pm}$are frequencies that give $n_{w}=0$ [Eqs. (5), (6), (13)].

$$
\Omega_{ \pm}=1 / 2\left( \pm \Omega_{b}+\sqrt{4+\Omega_{b}^{2}}\right) .
$$

As defined before, $\Omega_{b}=e^{2} b /\left(2 \pi^{2} \varepsilon_{0} \varepsilon_{b} \hbar \omega_{p}\right)$, where $b=$ $0.37 \AA^{-1}$ for pyrochlore [22] and plasmon frequency is given by $[17]$

$$
\omega_{p}=\sqrt{\frac{4 \alpha}{3 \pi}} \frac{E_{\mathrm{F}}}{\hbar}
$$

with $\alpha=\frac{e^{2}}{\hbar v_{\mathrm{F}} \varepsilon_{0} \varepsilon_{b}}$ and $v_{\mathrm{F}}=4 \times 10^{7} \mathrm{~cm} / \mathrm{s}$. [22]

From Fig. 2(a), it is important to point out that we may have a propagating wave even at frequencies smaller than plasmon frequency $(\Omega<1)$, in the shaded region II, which is in contrast with NM where an EM wave can propagate if $\Omega>1$ [see inset of Fig. 2(a)]. As shown in the inset of Fig. 2(a), the refractive indices of WSM and NM differ only near $\Omega \simeq 1$. At $\Omega \gg 1$, they both converge to the value of $n \approx \sqrt{\varepsilon_{b}}$. It is important to note that the negative solution of Eq. (13) $\left(n_{w}^{-}\right)$is assigned to have propagating wave toward positive $z$ direction in the region II, which will be shown later.

Let us calculate the reflection and transmission spectra. In NM with applied external magnetic field, the polarization of EM wave undergoes rotation as it enters the material if the direction of propagation is parallel to the direction of applied external magnetic field making the wave polarization not linear. In our case of WSM, we choose the propagation direction of the purely TM wave $\left(E_{y}=0\right)$ to be perpendicular to the "effective applied magnetic field", which is in the direction of the $\mathbf{b}=b \hat{\mathbf{y}}$. Therefore, we expect no rotation of polarization and the wave polarization keeps linear as TM wave. This fact can also be deduced from the vanishing $\varepsilon_{x y}$ and $\varepsilon_{z y}$. As shown in Fig. 1(b), the incident, reflected, and transmitted electric fields $\mathbf{E}_{i}, \mathbf{E}_{r}$ and $\mathbf{E}_{t}$ can be written as

$$
\begin{aligned}
& \mathbf{E}^{i}(z)=\left(\cos \theta_{i}, 0,-\sin \theta_{i}\right) E_{0}^{i} \exp \left(i k_{v z} z\right), \\
& \mathbf{E}^{r}(z)=\left(-\cos \theta_{i}, 0,-\sin \theta_{i}\right) E_{0}^{r} \exp \left(-i k_{v z} z\right), \\
& \mathbf{E}^{t}(z)=\left(\cos \theta_{t}, 0,-\sin \theta_{t}\right) E_{0}^{t} \exp \left(i k_{w z}^{ \pm} z\right),
\end{aligned}
$$

with $k_{v z}=(\omega / c) \cos \theta_{i}$ and $k_{w z}^{ \pm}=\omega\left(n_{w}^{ \pm} / c\right) \cos \theta_{t}$. The angles $\theta_{i}$ and $\theta_{t}$ are related each other by the Snell's law $\sin \theta_{i}=n_{w}^{ \pm} \sin \theta_{t}$. The magnetic fields in the $y$ direction can be obtained from the relations $H_{y}^{i, r}=i \omega \int \varepsilon_{0} E_{x}^{i, r} d z$ and $H_{y}^{t}=i \omega \int D_{x}^{t} d z$, where $D_{x}^{t}=\varepsilon_{1} E_{x}^{t}+i \varepsilon_{2} E_{z}^{t}$ is obtained from Eq. (7). Then, the magnetic fields can be written as

$$
\begin{aligned}
\mathbf{H}^{i}(z)= & \frac{\omega \varepsilon_{0}}{k_{v z}}\left(0, \cos \theta_{i}, 0\right) E_{0}^{i} \exp \left(i k_{v z} z\right), \\
\mathbf{H}^{r}(z)= & \frac{\omega \varepsilon_{0}}{k_{v z}}\left(0, \cos \theta_{i}, 0\right) E_{0}^{r} \exp \left(-i k_{v z} z\right), \\
\mathbf{H}^{t}(z)= & \frac{\omega}{k_{w z}^{ \pm}}\left(0, \varepsilon_{1} \cos \theta_{t}-i \varepsilon_{2} \sin \theta_{t}, 0\right) E_{0}^{t} \\
& \times \exp \left(i k_{w z}^{ \pm} z\right) .
\end{aligned}
$$

After defining the EM fields in both media, we can write down boundary conditions of the EM wave at incidence surface $(z=0)$ as follows,

$$
E_{0}^{i} \cos \theta_{i}-E_{0}^{r} \cos \theta_{i}=E_{0}^{t} \cos \theta_{t}
$$

and

$$
\begin{aligned}
& \frac{\omega \varepsilon_{0}}{k_{v z}}\left(E_{0}^{i} \cos \theta_{i}+E_{0}^{r} \cos \theta_{i}\right) \\
& =\frac{\omega}{k_{w z}^{ \pm}}\left(\varepsilon_{1} E_{0}^{t} \cos \theta_{t}-i \varepsilon_{2} E_{0}^{t} \sin \theta_{t}\right),
\end{aligned}
$$

where Eqs. (22) and (23) describe the continuity for the tangential components of electric fields and magnetic fields at $z=0$, respectively. Reflection coefficient $r=E_{0}^{r} / E_{0}^{i}$ and transmission coefficient $t=E_{0}^{t} / E_{0}^{i}$ are given by

$$
r=1-t \frac{\cos \theta_{t}}{\cos \theta_{i}}
$$

and

$$
t=\frac{2 k_{w z}^{ \pm} \varepsilon_{0} \cos \theta_{i}}{k_{v z}\left(\varepsilon_{1} \cos \theta_{t}-i \varepsilon_{2} \sin \theta_{t}\right)+k_{w z}^{ \pm} \varepsilon_{0} \cos \theta_{t}} .
$$




\section{RESULTS AND DISCUSSION}

In Fig. 3, we plot the reflection probability defined by $R=|r|^{2}$ as a function of $\theta_{i}$ for region I and III (that is $\Omega=0.3$ and 1.2 , respectively). Fig. $3(\mathrm{a})$ shows $R$ if we use $n_{w}^{+}$and Fig. $3(\mathrm{~b})$ shows $R$ if we use $n_{w}^{-}$. From Fig. 3, we can see that the incident EM wave will be totally reflected $R=1$ for all $\theta_{i}$ for both $n_{w}^{ \pm}$as shown in Fig. 3(a) and (d), due to the purely imaginary $n_{w}^{ \pm}$ given in Fig. 2(a). The $\mathbf{E}^{t}$ is decaying inside WSM, hence no transmitted energy into WSM. The most interesting case is region II, where we predict that WSM acquires a negative refractive index. In region II, we have a real $n_{w}^{ \pm}$, which means that the wave propagation inside WSM is allowed, even though the wave frequency is smaller than the plasmon frequency.

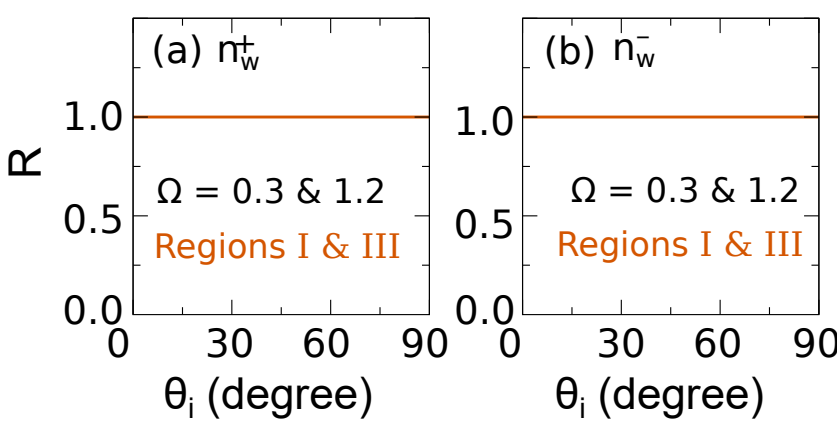

FIG. 3. The reflection probability (R) of the EM wave in a WSM for region I $[\Omega=0.3]$ and region III $[\Omega=1.2]$ for (a) $n_{w}^{+}$and $(\mathrm{b}) n_{w}^{-}$.

Normally, we use $n_{w}^{+}$which gives a positive value of $k_{w z}^{ \pm}$ because the transmitted wave propagates toward positive $z$ direction [see Eq. (18)]. However, $n_{w}^{+}$for the transmitted wave in region II gives an unphysical $R>1$, which means that at the point of incidence there is a flux of energy coming from the WSM side. We can infer from Eqs. (24) and (25) that $R>1$ if $n_{w}^{+}$is selected for region II. The reflection coefficient $r$ can be written as

$$
\begin{aligned}
r & =\frac{A-B-i C}{A+B-i C} \\
& =r_{1}+i r_{2}
\end{aligned}
$$

where $A=\varepsilon_{1} \cos \theta_{i} \cos \theta_{t}, \quad B=n_{w}^{ \pm} \varepsilon_{0} \cos ^{2} \theta_{t}, C=$ $\varepsilon_{2} \cos \theta_{i} \sin \theta_{i}$. The reflection probability can be obtained from $R=r_{1}^{2}+r_{2}^{2}$, where we define

$$
\begin{aligned}
& r_{1}=\frac{(A+B)(A-B)+C^{2}}{(A+B)^{2}+C^{2}} \\
& r_{2}=\frac{2 C B}{(A+B)^{2}+C^{2}} .
\end{aligned}
$$

$R>1$ if either $r_{1}>1$ or $r_{2}>1$. Let us investigate the case of $r_{1}$. From Eq. (28), we can define the requirement in order to have $r_{1}<1$ giving us physically sound $R<1$, otherwise we will have unphysical $R>1$,

$$
|A-B|<|A+B|
$$

or

$\left|\varepsilon_{1} \cos \theta_{i}-n_{w}^{ \pm} \varepsilon_{0} \cos \theta_{t}\right|<\left|\varepsilon_{1} \cos \theta_{i}+n_{w}^{ \pm} \varepsilon_{0} \cos \theta_{t}\right|$.

To better visualize Eq. (31), we plot $|A-B|$ and $|A+B|$ as a function of $\Omega$. From Fig. 4(a), where $n_{w}^{+}$ is selected, $|A-B|>|A+B|$ in region II, which does not fulfill Eq. (31) giving the unphysical $R>1$. On the other hand, from Fig. 4(b), where $n_{w}^{-}$is selected, $|A-B|<|A+B|$ in region II, which fulfills Eq. (31) and we can have physically correct $R<1$. This negative solution $\left(n_{w}^{-}\right)$should be selected only for region II, because if we apply $n_{w}^{-}$to region IV, we have an unphysical $R>1$, which is shown by Fig. 4(b), in which $|A-B|>|A+B|$ for region IV. We argue later that the reason why $n_{w}^{-}$is selected in region II for having transmitted wave toward positive $z$-direction, is due to the energy conservation.

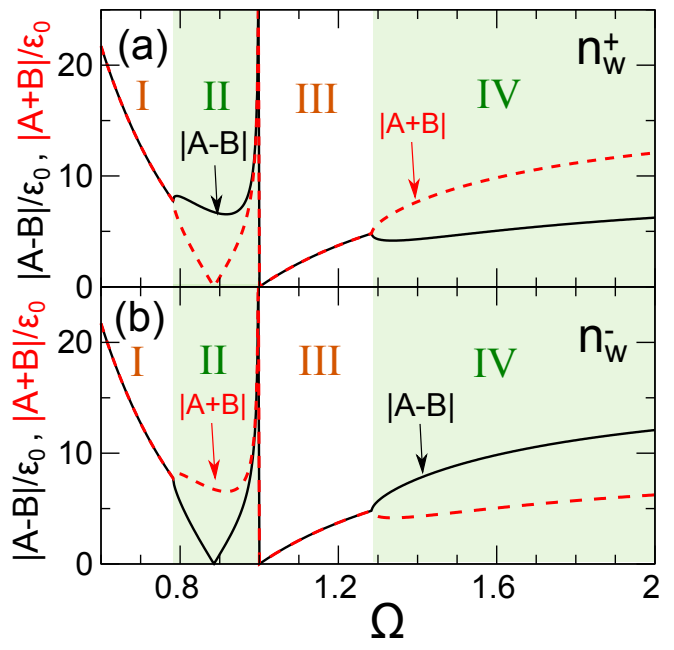

FIG. 4. $|A-B|$ and $|A+B|$ as a function of $\Omega$ if we use (a) $n_{w}^{+}$and (b) $n_{w}^{-}$. In region II, $n_{w}^{-}$is selected to fulfill Eq. (31), while in region IV, $n_{w}^{+}$is selected. Otherwise, we will have unphysical $R>1$ in both region.

The negative refractive index of WSM in region II will cause the wave refracted negatively, which means that the refracted angle $\theta_{t}$ is negative. The refractive index also means that the wave vector of transmitted wave $\left(k_{w z}^{-}\right)$ is negative. [28-31] The negative wave vector does not mean that the transmitted wave propagates backward, which violates the conservation of energy. The direction of propagation is better determined by the direction of the Poynting vector. By using Eqs. (18) and (21) at $z=0$, the power per unit cross section transmitted in the direction of $z$ can be expressed as

$$
\begin{aligned}
I_{t} & =\mathbf{S}_{t} \cdot \hat{\mathbf{z}} \\
& =\frac{1}{2} \operatorname{Re}\left[\mathbf{E}^{t}(0) \times \mathbf{H}^{* t}(0)\right] \cdot \hat{\mathbf{z}} \\
& =\frac{c|t|^{2}\left|E_{0}^{i}\right|^{2}}{2 n_{w}^{ \pm}} \varepsilon_{1} \cos \theta_{t},
\end{aligned}
$$


In order to have transmitted power propagate toward positive $z$ direction, Eq. (32) should have a positive value. Since $\varepsilon_{1}<0$ in region II [Eq. (5)], while $|t|^{2},\left|E_{0}^{i}\right|^{2}$, and $\cos \theta_{t}>0, n_{w}^{ \pm}$has to be negative $\left(n_{w}^{-}\right)$in order to have $I_{t}>0$. On the other hand, $n_{w}^{+}$is selected in region IV, because $\varepsilon_{1}>0$. We refer the transmitted wave as backward wave because the transmitted wave vector points towards negative $z$-direction shown by Fig. 5, otherwise it is forward wave. In short, the negative refraction is needed for the propagation of the EM wave with frequency smaller than the plasmon frequency to conserve energy.

To show the negative refraction more explicitly, we calculate the tangential component of the transmitted Poynting vector with respect to the interface. The tangential component of Poynting vector is given by,

$$
\begin{aligned}
\mathbf{S}_{t} \cdot \hat{\mathbf{x}}= & =\frac{1}{2} \operatorname{Re}\left[\mathbf{E}^{t}(0) \times \mathbf{H}^{* t}(0)\right] \cdot \hat{\mathbf{x}} \\
& =\frac{c|t|^{2}\left|E_{0}^{i}\right|^{2}}{2\left(n_{w}^{ \pm}\right)^{2}} \varepsilon_{1} \sin \theta_{i} .
\end{aligned}
$$

Because at region II, $\varepsilon_{1}<0$ and all other terms are positive, then $\mathbf{S} \cdot \hat{\mathbf{x}}<0$, which means that we have negative refraction. Therefore, at region II, we expect the light is transmitted as backward wave with negative refraction shown by Fig. 5 .

It is also interesting to compare our case with hyperbolic metamaterial. The negative refraction phenomenon in WSM is similar to hyperbolic metamaterials, where we can obtain negative refraction without having negative magnetic permeability. In hyperbolic metamaterials, due to the anisotropy of its dielectric tensor with respect to crystal axis, where the parallel and perpendicular component of dielectric tensor are opposite sign $\left(\overline{\bar{\varepsilon}}=\varepsilon_{\perp} \hat{\mathbf{x}} \hat{\mathbf{x}}+\varepsilon_{\|}[\hat{\mathbf{y}} \hat{\mathbf{y}}+\hat{\mathbf{z}} \hat{\mathbf{z}}]\right.$, with $\left.\varepsilon_{\perp}<0, \varepsilon_{\|}>0\right)$, the light can be refracted negatively as a forward wave. [32, 33] This refraction phenomenon can also take place in bulk Rashba system, which can act as hyperbolic metamaterial at certain frequency range. [34] Therefore, due to the forward transmitted wave, in hyperbolic metamaterial the negative refraction can take place without having negative effective refractive index. This situation is different from our case for WSM, where the negative refraction takes place with backward transmitted wave, similar to Veselago medium.

If we use $\Omega_{b}=0.5$ we have $\omega_{p}=800 \mathrm{THz}$ and the corresponding region II can be found within $(625 \leq \omega \leq 800 \mathrm{THz})$. If we use $\omega_{p}=9 \mathrm{THz}$ which is measured in experiment, $[22] \Omega_{b}=44$ and the corresponding region II can be found within $(0.2 \leq \omega \leq 9 \mathrm{THz})$. by

Using Eq. (32), the transmission probability $T$ is given

$$
T=\frac{I_{t}}{I_{i}}=\frac{1}{n_{w}^{ \pm}} \frac{\varepsilon_{1}}{\varepsilon_{0}} \frac{\cos \theta_{t}}{\cos \theta_{i}}|t|^{2},
$$

where $I_{i}=(c / 2)\left|E_{0}^{i}\right|^{2} \varepsilon_{0} \cos \theta_{i}$ is the incident intensity.

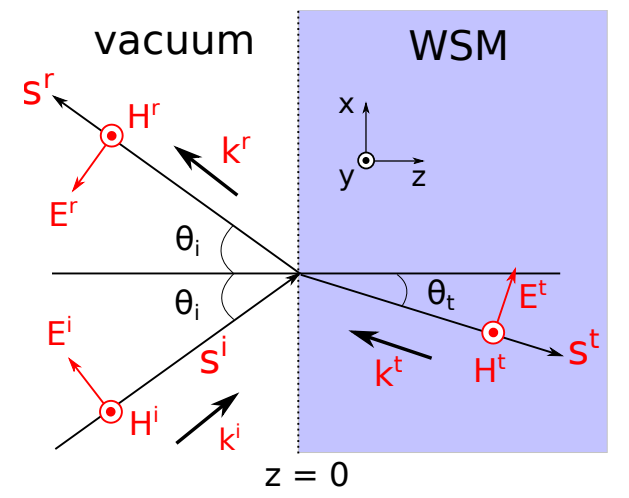

FIG. 5. The negative refraction in WSM. $\mathbf{S}_{t}$ is the transmitted Poynting vector. $\mathbf{k}_{i}, \mathbf{k}_{r}, \mathbf{k}_{t}$ are incident, reflected and transmitted wave vectors, respectively.
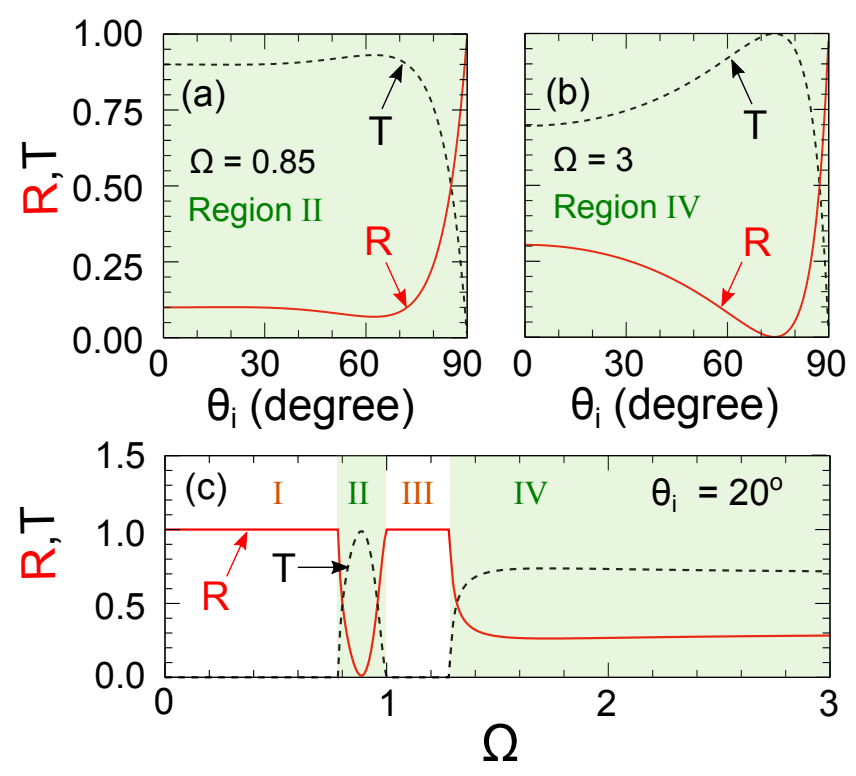

FIG. 6. The $R$ and $T$ spectra as a function of $\theta_{i}$ shown as solid and dashed line, respectively, for (a) region II $(\Omega=0.85)$, (b) region IV $(\Omega=3)$. In (a) the negative solution of $n_{w}$ is used, while in (b) the positive one is used. In both cases, $R+T=1$. (c) The $R$ and $T$ spectra as a function of $\Omega$ with fixed $\theta_{i}=20^{\circ}$ shown as solid and dashed line, respectively. In shaded regions II and IV, the wave is transmitted. However, only in region II we expect that negative refraction could occur.

The reflection probability $R$ is given by $R=|r|^{2}$. In Figs. 6(a) and 6(b) we show the $R$ and $T$ spectra for region II $(\Omega=0.85)$ and region IV $(\Omega=3)$, where the EM wave propagation is allowed. In the case of region II, we adopt the $n_{w}^{-}$, while in the case of region IV, we adopt $n_{w}^{+}$. In region IV, the WSM acts as a NM for $\Omega>1$. Figure $6(\mathrm{~b})$ shows $R=0$ at $\theta_{i}=\arctan n_{w}$, which corresponds to the Brewster angle. In both cases, we found $R+T=1$. In Fig. 6(c), we plot the $R$ and $T$ spectra as a function of $\Omega$ at a fixed incident angle $\theta_{i}=20^{\circ}$. In region II, we expect that the negative refraction can take place. In NM, all EM wave is reflected in the region 

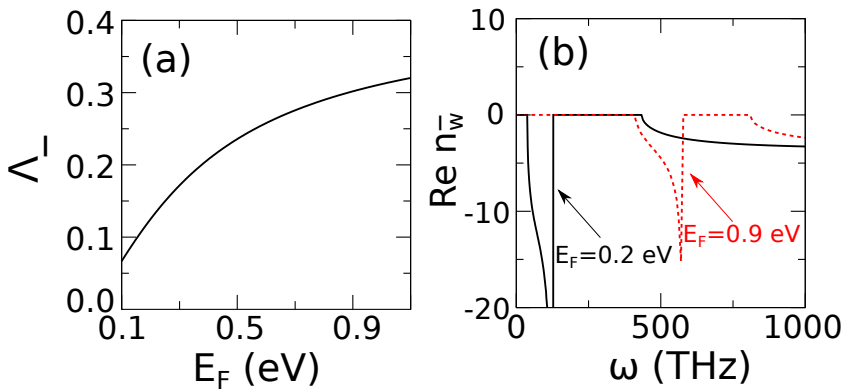

FIG. 7. (a) The $\Lambda_{-}$as a function of $E_{\mathrm{F}}$. (b) The real part of $n_{w}^{-}$for $E_{\mathrm{F}}=0.2 \mathrm{eV}$ and $0.9 \mathrm{eV}$.

II due to the imaginary transmitted wave vector. The region II of WSM, the $R$ gradually decreases with increasing $\Omega$ because the transmitted wave vector acquires real value, which signifies the transmission of the incident wave to WSM. After reaching the minimum of $R$ at $\Omega=0.9$, the reflection probability increases gradually up to $R=1$ at $\Omega=1$, above which the transmitted wave vector has only imaginary value that makes $T=0$. It is important to note that the negative refraction in WSM occurs only in region II, which has frequency range close to $\omega_{p}$, which can be seen in Figs. 2 and 6(c). Because $\omega_{p}$ depends on $E_{\mathrm{F}}$ [See Eq. (15)], by controlling the $E_{\mathrm{F}}$, we can control the frequency, where negative refraction occurs, which will be discussed as below.

It is also useful to have a parameter that gives us information whether or not we have negative refraction for a given $E_{\mathrm{F}}$. By using Eq. (15), the frequency range of region II, where we expect the negative refraction, can be rewritten as $\Lambda_{-}<\frac{\hbar \omega}{E_{\mathrm{F}}}<\sqrt{\frac{4 \alpha}{3 \pi}}$, where $\Lambda_{-}=\sqrt{\frac{4 \alpha}{3 \pi}} \Omega_{-}$ and $\sqrt{\frac{4 \alpha}{3 \pi}}=0.423$. $\Omega_{-}$is given by Eq. (14). $\Lambda_{-}$is plot- ted in Fig. 7(a) as a function of $E_{\mathrm{F}}$. Hence, by taking ratio of EM wave energy $(\hbar \omega)$ and $E_{\mathrm{F}}$, we can predict whether the negative refraction occurs by using Fig. 7(a). In Fig. $7(\mathrm{~b})$, we plot the $n_{w}^{-}$as a function of frequency $(\omega)$ in a real unit for $E_{\mathrm{F}}=0.2 \mathrm{eV}$ and $0.9 \mathrm{eV}$. Increasing $E_{\mathrm{F}}$ will shift the region II and region IV to higher frequency. The frequency range of region II monotonically increases with increasing $E_{\mathrm{F}}$. Note that $\alpha$ depends on $v_{\mathrm{F}}$ [see Eq. (15)].

\section{CONCLUSION}

In conclusion, we have shown theoretically that negative refraction can occur in the WSM, which is justified from its reflection spectra. The refractive index of WSM is negative at a specific frequency range close to the plasmon frequency. The negative refractive index is required for the propagation of TM EM wave with frequency smaller than the plasmon frequency in the direction perpendicular to the separation of Weyl nodes to conserve the energy and to obtain the physically correct solution. We suggest that by using only the WSM, it is not necessary to make a complicated structure of metamaterials to obtain negative refraction. It would be desired if the phenomenon could be measured in future experiments.

\section{ACKNOWLEDGMENTS}

M.S.U is supported by the MEXT scholarship, Japan. A.R.T.N. acknowledges the Leading Graduate School in Tohoku University. R.S. acknowledges JSPS KAKENHI Grant Numbers JP25107005 and JP25286005.
[1] V. G. Veselago, Phys. Usp. 10, 509 (1968).

[2] E. Cubukcu, K. Aydin, E. Ozbay, S. Foteinopoulou, and C. M. Soukoulis, Nature 423, 604 (2003).

[3] J. B. Pendry, Phys. Rev. Lett. 85, 3966 (2000).

[4] T. Habe and M. Koshino, Phys. Rev. B 91, 201407 (2015).

[5] V. V. Cheianov, V. Fal'ko, and B. Altshuler, Science 315, 1252 (2007).

[6] D. R. Smith, J. B. Pendry, and M. C. K. Wiltshire, Science 305, 788 (2004).

[7] J. Lu, T. M. Grzegorczyk, Y. Zhang, J. Pacheco Jr, B.-I. $\mathrm{Wu}$, J. A. Kong, and M. Chen, Opt. Express 11, 723 (2003).

[8] D. Ziemkiewicz and S. Zielińska-Raczyńska, J. Opt. Soc. Am. B 32, 1637 (2015).

[9] V. M. Shalaev, Nat. Photon. 1, 41 (2007).

[10] A. Boltasseva and V. M. Shalaev, Metamaterials 2, 1 (2008).

[11] W. J. Padilla, D. N. Basov, and D. R. Smith, Mater. Today 9, 28 (2006).
[12] J. Valentine, S. Zhang, T. Zentgraf, E. Ulin-Avila, D. A. Genov, G. Bartal, and X. Zhang, Nature 455, 376 (2008).

[13] A. Ishikawa, T. Tanaka, and S. Kawata, Phys. Rev. Lett. 95, 237401 (2005).

[14] H. Moser, B. Casse, O. Wilhelmi, and B. Saw, Phys. Rev. Lett. 94, 063901 (2005).

[15] F. Bilotti, A. Toscano, and L. Vegni, IEEE Trans. Antennas. Propag. 55, 2258 (2007).

[16] P. V. Parimi, W. T. Lu, P. Vodo, and S. Sridhar, Nature 426, 404 (2003).

[17] J. Hofmann and S. Das Sarma, Phys. Rev. B 93, 241402 (2016).

[18] A. A. Burkov and L. Balents, Phys. Rev. Lett. 107, 127205 (2011).

[19] M. M. Vazifeh and M. Franz, Phys. Rev. Lett. 111, 027201 (2013).

[20] M. Koshino and I. F. Hizbullah, Phys. Rev. B 93, 045201 (2016).

[21] Y. Ominato and M. Koshino, Phys. Rev. B 91, 035202 (2015). 
[22] A. B. Sushkov, J. B. Hofmann, G. S. Jenkins, J. Ishikawa, S. Nakatsuji, S. Das Sarma, and H. D. Drew, Phys. Rev. B 92, 241108 (2015).

[23] A. A. Zyuzin and V. A. Zyuzin, Phys. Rev. B 92, 115310 (2015).

[24] A. G. Grushin, Phys. Rev. D 86, 045001 (2012).

[25] A. A. Zyuzin and A. A. Burkov, Phys. Rev. B 86, 115133 (2012).

[26] A. A. Zyuzin, S. Wu, and A. A. Burkov, Phys. Rev. B 85, 165110 (2012).

[27] P. Hosur and X. Qi, C. R. Phys. 14, 857 (2013).
[28] B. E. A. Saleh and M. C. Teich, Fundamentals of photonics (Wiley, New York, 1991).

[29] D. R. Smith and N. Kroll, Phys. Rev. Lett. 85, 2933 (2000).

[30] S. A. Ramakrishna, Rep. Prog. Phys. 68, 449 (2005).

[31] J. Woodley and M. Mojahedi, J. Opt. Soc. Am. B 23, 2377 (2006).

[32] A. Poddubny, I. Iorsh, P. Belov, and Y. Kivshar, Nat. Photon. 7, 948 (2013).

[33] P. A. Belov, Microw. Opt. Technol. Lett. 37, 259 (2003).

[34] J. Shibata, A. Takeuchi, H. Kohno, and G. Tatara, J. Phys. Soc. Japan 85, 033701 (2016). 